\title{
Withaferin A (WIT) Interaction with beta-Tubulin to Promote Tubulin Degradation: In Silico Study
}

\section{Mehdi Nabati ${ }^{1, \otimes}$, Elham Pournamdari², Yahya Dashti-Rahmatabadi³, Saman Sarshar ${ }^{4}$}

Received: 20 February 2020 / Accepted: 02 M arch 2020 / Published Online: 15 M arch 2020

(c) SAM I Publishing Company (SPC) 2020

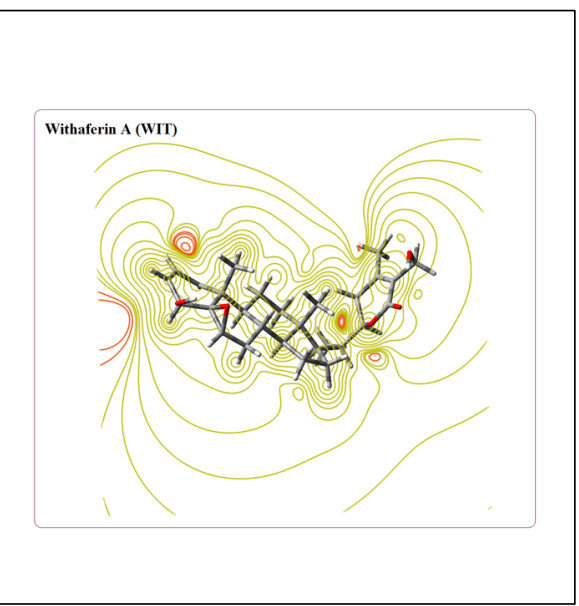

\begin{abstract}
The main purpose of present study is evaluation of structural and medicinal properties for W ithaferin A (WIT) using density functional theory (DFT) method. All studies are done via computational chemistry methods using Gaussian 03 and M olegro Virtual Docker (MVD) software packages and SwissADME web-based tool. Molecular structure of WIT was optimized at the B3LYP/6$311+G(d, p)$ theoretical level of DFT. The reactivity and stability properties of the optimized molecule were explored via global reactivity indices. Calculating the reactivity indices using energies of frontier molecular orbitals (FMOs) showed that WIT is stable against the oxidizing agents in the cell and has low reactivity against the biomolecules. On the other hand, the docking analysis data indicated the steric interactions play important role in WIT binding to beta-Tubulin via the residues Tyr224, Cys12, GIn11, Asn101, Gly143, GIn15, Gly144, Asn206, Gly142, and Asp179.
\end{abstract}

Keywords: In silico; M olecular docking; M olecular simulation; Tubulin; Withaferin A.

\section{Introduction}

Withaferin A (WIT), an important natural compound, is extracted from the Indian medicinal plant Withania somnifera. This plant has been used for over 3000 years in Unani and Indian medical systems. Also, its extracted medicinal

\footnotetext{
$\triangle$ Corresponding author.

E-mail address: mnabati@ymail.com (M . Nabati)

${ }^{1}$ Research and Development Department, Shari Pharmaceutical Company, Tehran, Iran
}

compound has been used in ayurvedic medicine with multiple pharmacological activities containing immune-modulatory, anti-diabetic, cardioprotective, anti-inflammatory, antimetastasis, anti-angiogenesis and anticarcinogenic properties [1-6]. Several active

\footnotetext{
2 Department of Science, Islamshahr Branch, Islamic Azad University, Islamshahr, Iran

3 Science and Research Branch, Islamic Azad University, Tehran, Iran

4 Physics Department, Faculty of Science, Lorestan University, Khorramabad, Iran
} 
groups, such as hydroxyl, epoxide ring, carbonyl, double bond and lactone ring are in the molecular structure of WIT. These active groups cause strong interactions of the molecule with amino acid residues of different targets, such as Vimentin, IKB kinase $\beta$ (IKK $\beta$ ), Annexin II and $\mathrm{N}$-terminus of heat shock protein 90 (HSP90) [7-12]. Recently, the researchers have found out the anti-carcinogenic properties of the title medicinal compound is due to its interaction with beta Tubulin. In 2014, Antony and co-workers [13] reported that WIT inhibits beta-Tubulin (the major component of the eukaryotic cytoskeleton) activity by predicting the ligand-receptor complex formation of WIT binding to Cys303 of beta-Tubulin. In 2019, Yang and coworkers [14] conducted an in-depth study of the interactions between WIT and Tubulin. Their studies showed that the molecule under study forms covalent bonds with residues Cys303 and Cys239 of the protein. Unfortunately, they did not provide much information about the exact structural and molecular drug-receptor interactions, such as hydrogen bond, ionic and steric interactions. Analysis of the exact molecular mechanisms involved in interaction of WIT with beta-Tubulin proteins is the main aim of the present research work. These studies are done using computational chemistry and molecular docking methods. Moreover, the pharmacokinetic behavior and biological attributes of the titled medicinal molecular structure are determined using SwissADM E web tool.

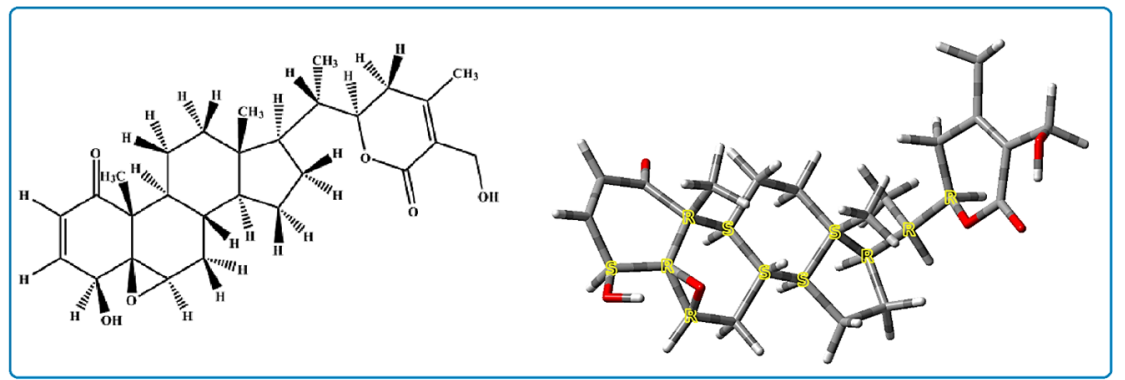

Fig. 1. The optimized molecular structure of Withaferin A.

\section{Materials and Methods}

In silico study is a good technique to analyze some properties and applications of a chemical compound which are not easily investigated via experimental methods [15-25]. These studies are mainly done by computer software packages [26]. As mentioned above, the important goal of the present study is analysis the interaction of Withaferin A (WIT) with beta-Tubulin protein. To this aim, the molecular structure of the studied compound was optimized using Gaussian 03 software via B3LYP/6-311+HG(d,p) level of theory [27]. Its stability, reactivity and electronic properties were investigated via calculating the global reactivity indices. The frontier molecular orbitals (FMOs) energies were used to compute the titled indices [28-31]. In the next step, the optimized molecular structure was embedded into the active site of the protein. This work was done using Molegro Virtual Docker (MVD) software. From analysis of molecular docking data, the exact types of interactions of the ligand-protein complex were evaluated. Finally, the physicochemical and ADME properties of the WIT natural compound were predicted via www.swissadme.ca web-based tool.

\section{Results and discussion}

\section{Structural Properties of Withaferin A}

Withaferin $A$ is a $28-C$ steroidal lactone that its backbone contains three cyclohexane rings and one cyclopentane ring (Fig. 1). The alpha, betaunsaturated ketone-containing ring, the epoxidecontaining ring, and alpha, beta-unsaturated 
ketone-containing ring are more reactive parts of the molecule [13]. The said molecular structure was optimized using DFT methods. After each computation, the calculated bond lengths of the molecular structure were compared with the corresponding empirical data. The highest correlation coefficient of the theoretical-empirical bond lengths dependence was seen for computations at the B $3 L Y P / 6-311+H G(d, p)$ level of theory. The electronic energy of the optimized molecular structure is $-41444 \mathrm{eV}$. The optimized molecule has high stability with the dipole moment of 5.8638 Debye. Fig. 2 indicates the dependence between the theoretical and experimental bond lengths of the WIT medicinal compound. This dependency is shown by the equation of $y=1.0475 x-0.0676$. The higher correlation coefficient $\left(R^{2}=0.9921\right)$ for this equation shows a great convergence. Therefore, the $B 3 L Y P / 6-311+H(d, p)$ level of theory is a good method to compute the electronic properties of the title compound.

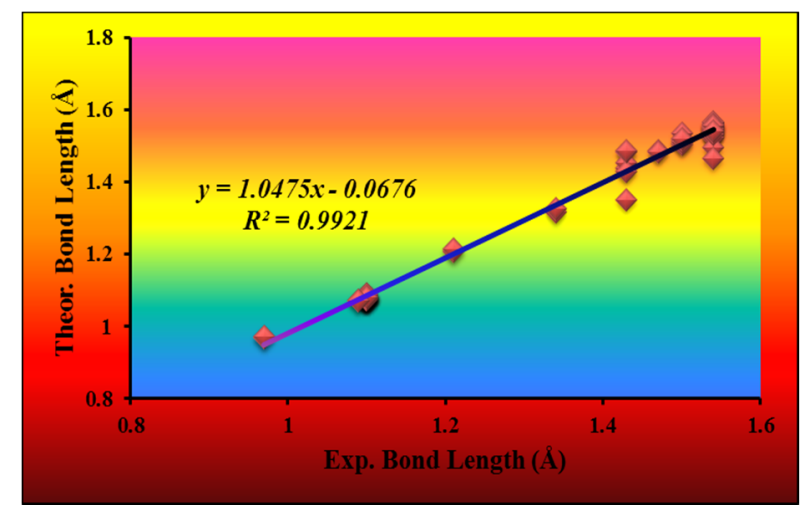

Fig. 2. The experimental and theoretical bond lengths relationship of Withaferin $A$.

\section{Stability and Reactivity of Withaferin A}

Calculating the global reactivity indices is a good way to study the stability and reactivity properties of a chemical compound [28-32].The global reactivity descriptors like energy gap $\left(E_{g}\right)$, ionization potential (IP), electron affinity (EA), chemical hardness $(\eta)$, chemical softness (S), electronegativity $(\chi)$, electronic chemical potential $(\mu)$ and electrophilicity index $(\omega)$ can be obtained from the energies of the frontier orbitals. These reactivity indices are achieved by following formulas [33]:

$$
\begin{aligned}
& E_{g}=E_{\text {LUMO }}-E_{\text {HOMO }} \\
& I P=-E_{\text {HOMO }} \\
& E A=-E_{\text {LUMO }} \\
& \eta=\frac{\left(\varepsilon_{\text {LUMO }}-\varepsilon_{\text {HOMO }}\right)}{2} \\
& \chi=\frac{-\left(\varepsilon_{\text {LUMO }}+\varepsilon_{\text {HOMO }}\right)}{2} \\
& \mu=\frac{\left(\varepsilon_{\text {LUMO }}+\varepsilon_{\text {HOMO }}\right)}{2} \\
& \omega=\frac{\mu^{2}}{2 \eta} \\
& S=\frac{1}{\eta}
\end{aligned}
$$

Table 1: Global reactivity indices of Withaferin A

\begin{tabular}{ll}
\hline Parameter & Energy value $(\mathrm{eV})$ \\
\hline HOMO & -10.29 \\
LUMO & 2.77 \\
lonization Potential (IP) & 10.29 \\
Electron Affinity (EA) & 2.77 \\
Energy Gap $\left(\boldsymbol{E}_{\mathrm{g}}\right)$ & 13.06 \\
Electronegativity $(\boldsymbol{\alpha})$ & 3.26 \\
Chemical Potential $(\boldsymbol{\mu})$ & -3.26 \\
Chemical Hardness $(\boldsymbol{\eta})$ & 6.53 \\
Chemical Softness (S) & 0.153 \\
Electrophilicity index $(\boldsymbol{\omega})$ & 0.814 \\
\hline
\end{tabular}

Fig. 3 indicates the frontier molecular orbitals (FMOs) of the molecule under study. FMOs are called to the highest occupied molecular orbital (HOMO) and the lowest unoccupied molecular orbital (LUMO) [33]. The HOMO was made of atomic orbitals of lactone ring. In contrast, the atomic orbitals of the carbonyl containing ring. So, these rings prefer to make complex with the residues of the protein via nucleophilic and electrophilic interactions, respectively. On the other hand, the red, green and blue colors of the molecular electrostatic potential (MEP) graph relate to the negative, zero and positive charge 
densities, respectively. We can see the electronegative atoms have more charge density than other atoms. So, these atoms are more susceptible to interact with the electron deficient residues of the protein. The density of states (DOS) graph shows high energy gap (13.06 eV) between HOM O (-10.29 eV) and LUMO (2.77 eV). This high energy gap of the FM Os indicates the high stability of the compound. It can be deduced that the electronic transition can't happen in valence layer of the molecular orbitals. So, the molecule is stable against the oxidizing agents in the cell. The global reactivity indices have been listed in Table 1. The obtained data show that the compound has high chemical hardness $(6.53 \mathrm{eV})$ and low chemical softness $(0.153 \mathrm{eV})$ indices. So, it could be expected that the molecule has low reactivity against the biomolecules.

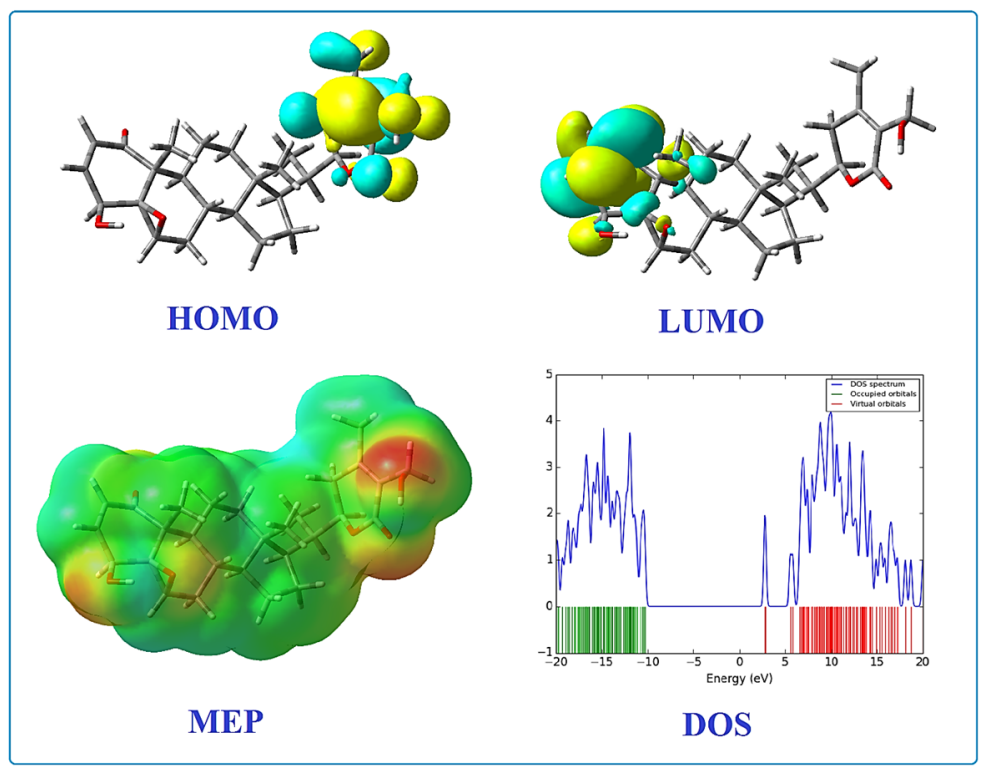

Fig.3. The FM Os, MEP and DOS of Withaferin A.

\section{Physicochemical Descriptors and ADME Parameters for Withaferin A}

In medicinal chemistry, ADME is the abbreviation for the absorption, distribution, metabolism and excretion properties. These properties are the key factors to predict the pharmaceutical activity [34]. Here, the ADME properties of the WIT compound was done using SwissADM E web-based tool.

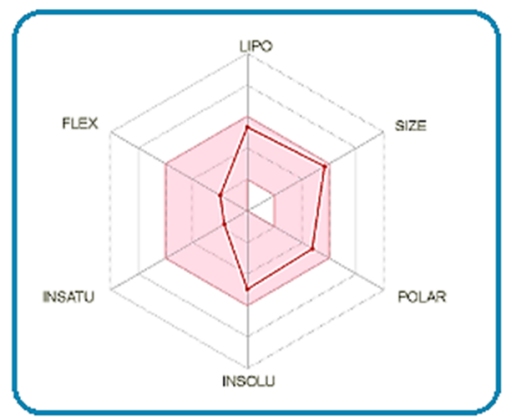

Fig. 4. Physicochemical properties graph of the compound Withaferin $\mathrm{A}$.
Fig. 4 shows the predicted physicochemical graph of the molecule under study. The oral bioavailability of a medicinal compound is identified via six key parameters: lipophilicity (-0.7 $<$ XLOGP3 < +5.0), size $(150 \mathrm{~g} / \mathrm{mol}<\mathrm{M}<500$ $\mathrm{g} / \mathrm{mol})$, polarity $\left(20 \AA^{2}<\mathrm{TPSA}<130 \AA^{2}\right)$, insolubility $(0<$ ESOL $<6)$, insaturation $(0.25<$ Fraction Csp3 < $1)$ and flexibility $(0<$ number of rotatable bonds < 9). The colored zone in this graph shows the suitable physicochemical space for oral bioavailability of a compound [35]. We can see the Fig. 6 indicates WIT has oral bioavailability. The evaluation of the compound's properties showed the lipophilicity (LogPo/w) of 3.83, molecular weight of $470.6 \mathrm{~g} / \mathrm{mol}$, TPSA of $96.36 \AA^{2}$, ESOL of 5.01, fraction Csp3 of 0.79 and3 rotatable bonds. The molecule under study shows the 
bioavailability score of 0.55 . On the other hand, the said molecule hasn't any activity as an inhibitor of the cytochrome P450 subunits. Fig. 5 shows the boiled egg graph of the WIT compound. The yellow-colored zone of the graph shows the permeation of the molecules through the bloodbrain barrier (BBB). In contrast, the white-colored zone of the boiled egg graph relates to the absorbed molecules by the gastrointestinal tract. The boiled egg graph indicates that WIT is absorbed by gastrointestinal tract. Also, the blue color of the molecule in this graph shows that it is effluated from the central nervous system (CNS) by the P-glycoprotein.

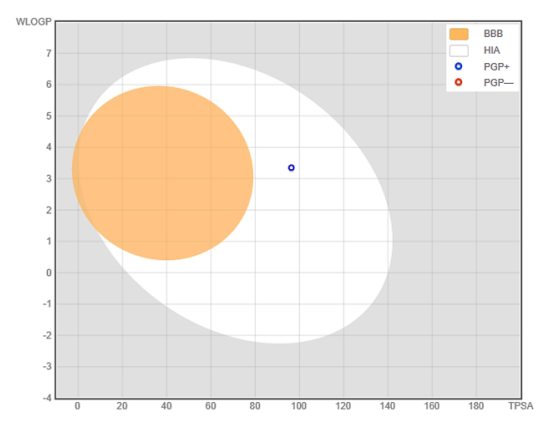

Fig. 5. Boiled egg graph of the compound Withaferin A.

\section{Molecular Docking Analysis of Ticagrelor-P2Y12 Complex}

Recent studies show that WIT inhibits betaTubulin activity via its binding to Cys303 of the said protein [13]. Here, complex formation between WIT and beta-Tubulin has been studied via molecular docking analysis. The Molegro Virtual Docker (MVD) program was used to perform the docking analysis of the ligand-receptor complex.

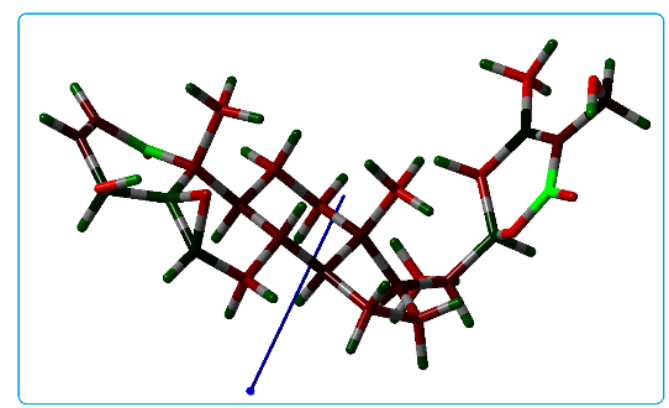

Fig. 6. Charge distribution and dipole moment vector of Withaferin $A$.
Fig. 6 indicates the charge distribution and dipole moment vector of WIT ( $X=1.7191, Y=1.3545$ and $Z=5.5821)$. The molecule under study showed the dipole moment of 5.8638 Debye. We can see all oxygen and carbon atoms except carbon atoms of the carbonyl functional groups have negative charges. So, the molecule prefers to interact with the electron poor residues.

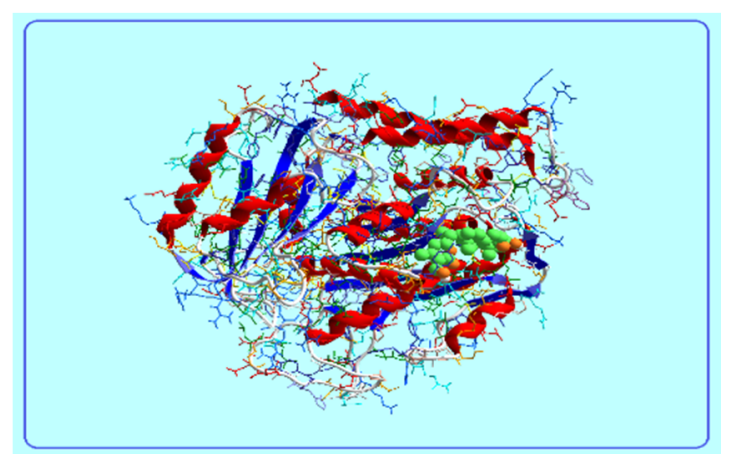

Fig. 7. Ligand Withaferin Aembedded in the active site of the beta-Tubulin.

Fig. 7 indicates that the ligand WIT is embedded in the active site of the beta- Tubulin. WIT made complex with the protein structure by Moldock score of -135.589 . So, WIT binding to beta-Tubulin is strong and is mainly done via the steric interactions (MolDock score of -152.219). The MolDock score of the hydrogen bonds in ligandreceptor complex was -4.505 . The internal ligand interactions weaken the binding between ligand and receptor. The internal torsional strain and steric interactions showed the MolDock scores of 4.203 and 27.859, respectively. The beta-Tubulin residues GIn15, Leu227, Asn228, Ile16, Gly142, Thr180, Asn101, Val171 Asn206, Asp179, Ser140, Tyr224, Cys12, Gly144, Gly143, Gly146, Thr145, Gln11, Asp69, Ala99, Gly100, Glu71, and Gly10 participated in steric interactions. In contrast, only the residues Gln15, Gly146 and Ala99 can make interaction with the molecule using hydrogen bond formation. Our studies showed the strongest interactions of WIT with beta-Tubulin relating to residues Tyr224, Cys12, Gln11, Asn101, Gly143, Gln15, Gly144, Asn206, Gly142, and Asp179. 


\section{Conclusion}

Investigating physicochemical, structural and electronic properties of Withaferin A (WIT) was the main objective of the present research work. Electronic properties prediction of the molecule was carried out using the computational chemistry methods. The molecular structure was optimized at the B3LYP/6-311+HG(d,p) level of theory. The energies of frontier molecular orbitals (HOM O and LUM O) were used to calculate the global reactivity indices. WIT is stable against the oxidizing agents in the cell with low reactivity against the biomolecules. The molecule-receptor Interactions

\section{References}

1. Balaguer FD, M ühlethaler T, Estévez-Gallego J, Calvo E, Giménez-Abián JF, Risinger AL, Sorensen EJ, Vanderwal CD, Altmann KH, Mooberry SL, Steinmetz M O. Crystal structure of the cyclostreptin-tubulin adduct: implications for tubulin activation by taxane-site ligands. Int. J. M ol. Sci. 2019;20:1392.

2. Bargagna-Mohan P, Hamza A, Kim YE, Ho YK, MorVaknin N, Wendschlag N, Liu J, Evans RM, Markovitz DM, Zhan CG, Kim KB. The tumor inhibitor and antiangiogenic agent withaferin $A$ targets the intermediate filament protein vimentin. Chem. Biol. 2007;14:623-634.

3. Buey RM, Calvo E, Barasoain I, Pineda O, Edler MC, Matesanz R, Cerezo G, Vanderwal CD, Day BW, Sorensen EJ, López JA. Cyclostreptin binds covalently to microtubule pores and lumenal taxoid binding sites. Nature Chem. Biol. 2007;3:117-125.

4. Drahl C, Cravatt BF, Sorensen EJ. Protein-reactive natural products. Angew. Chem. 2005;44:5788-5809.

5. Dumontet $\mathrm{C}$, Jordan M A. M icrotubule-binding agents: a dynamic field of cancer therapeutics. Nature Rev. Drug Discover. 2010;9:790-803.

6. Hamel E. An overview of compounds that interact with tubulin and their effects on microtubule assembly. In The Role of M icrotubules in Cell Biology, Neurobiology, and Oncology. Humana Press; 2008. pp. 1-19.

7. Falsey RR, Marron MT, Gunaherath GK, Shirahatti N, Mahadevan D, Gunatilaka AL, Whitesell L. Actin microfilament aggregation induced by withaferin $A$ is mediated annexin II. Nature Chem. Biol. 2006;2:33-38.

8. Fortin S, Lacroix J, Côté MF, Moreau E, Petitclerc É, René C. Quick and simple detection technique to assess indicate the main role of the beta-Tubulin residues containing Tyr224, Cys12, Gln11, Asn101, Gly143, Gln15, Gly144, Asn206, Gly142, and Asp179 in the ligand-receptor complex formation. The molecular analysis data showed the steric interactions in formation of the ligand-receptor complex. Finally, the ADME study showed that the said compound is non-toxic and has oral bioavailability.

\section{Acknowledgments}

The corresponding author is grateful to Dr. Hamideh Sabahno and Dr. Vida Bodaghi-Namileh for providing valuable suggestions to this work.

the binding of antimicrotubule agents to the colchicinebinding site. Biol. Proc. Online 2010;12:113-117.

9. Gambhir L, Checker R, Sharma D, Thoh M, Patil A, Degani M, Gota V, Sandur SK. Thiol dependent NF-kB suppression and inhibition of T-cell mediated adaptive immune responses by a naturally occurring steroidal lactone Withaferin A. Toxicol. Appl. Pharmacol. 2015;289:297-312.

10. Gigant B, Wang C, Ravelli RB, Roussi F, Steinmetz MO, Curmi PA, Sobel A, Knossow M. Structural basis for the regulation of tubulin by vinblastine. Nature 2005;435:519-522.

11. Gu M, Yu Y, Gunaherath GK, Gunatilaka AL, Li D, Sun D. Structure-activity relationship (SAR) of withanolides to inhibit Hsp90 for its activity in pancreatic cancer cells. Invest. New Drugs 2014;32:68-74.

12. Heyninck K, Lahtela-Kakkonen $M$, Van der Veken $P$, Haegeman G, Berghe WV. Withaferin A inhibits NFkappaB activation by targeting cysteine 179 in IKK 3 . Biochemical pharmacology. 2014;91:501-509.

13. Antony M L, Lee J, Hahm ER, Kim SH, Marcus Al, Kumari V, Ji X, Yang Z, Vowell CL, Wipf P, Uechi GT. Growth arrest by the antitumor steroidal lactone withaferin $A$ in human breast cancer cells is associated with downregulation and covalent binding at cysteine 303 of $\beta$ tubulin. J. Biol. Chem. 2014;289:1852-1865.

14. Yang J, Yan W, Li Y, Niu L, Ye H, Chen L. The natural compound Withaferin A covalently binds to Cys239 of $\beta$-tubulin to promote tubulin degradation. Mol. Pharmacol. 2019;96:711-719.

15. Nabati M, Bodaghi-Namileh V. M olecular modeling of 3-(1,3-dioxoisoindolin-2-yl) benzyl nitrate and its 
molecular docking study with phosphodiesterase- 5 (PDE5). Adv. J. Chem. A 2020;3:58-69.

16. Nabati M, Lohrasbi E, Sabahnoo H, Bodaghi-Namileh V, M azidi M, M ohammadnejad-M ehrabani H, Tavakkoli A, Gervand A. In silico study of metoclopramide as a small molecule of dopamine D2 receptor. Chem. Method. 2020;4:19-33.

17. Nabati M. Exploring molecular docking and electronic studies of [11C] LY2795050 as a novel antagonist tracer for positron emission tomography (PET) scan of the kappa ( $\kappa)$ and $\mathrm{mu}(\mu)$ opioid receptors (KOR and MOR). J. Med. Chem. Sci. 2020;3:22-34.

18. Nabati $M$, Bodaghi-Namileh V. In silico study of the active components (17 $\alpha$-ethinyl estradiol and segesterone acetate) of annovera as a novel vaginal contraceptive system by docking of their binding to estrogen and progesterone receptors. Iran. Chem. Commun. 2020;8:73-85.

19. Nazemi H, Mirzaei M, Jafari E. Antidepressant Activity of Curcumin by M onoamine Oxidase-A Inhibition. Adv. J. Chem. B 2019;1:3-9.

20. Ozkendir O, M irzaei M. Alkali metal chelation by 3hydroxy-4-pyridinone. Adv. J. Chem. B 2019;1:10-16.

21. Esfahani, A., Mirzaei, M. Flavonoid derivatives for monoamine oxidase-A inhibition. Adv. J. Chem. B 2019;1:17-22.

22. Alidoosti Z, Mirzaei M. Comparative examination of moclobemide, tranylcypromine, phenelzine and isocarboxazid for monoamine oxidase-A inhibition. Adv. J. Chem. B 2019;1:23-28.

23. Ariaei $\mathrm{S}$. Adsorptions of diatomic gaseous molecules ( $\mathrm{H} 2, \mathrm{~N} 2$ and $\mathrm{CO})$ on the surface of Li+@C16B8P8 fullerene-like nanostructure: computational studies. Adv. J. Chem. B 2019;1:29-36.

24. Soleimani M, Mirzaei M, Mofid MR, Khodarahmi G, Rahimpour SF. Lactoperoxidase inhibition by tautomeric propylthiouracils. Asian J. Green Chem. 2020;4:1-10.

25. Samadi Z, M irzaei M , Hadipour NL, Khorami SA. Density functional calculations of oxygen, nitrogen and hydrogen electric field gradient and chemical shielding tensors to study hydrogen bonding properties of peptide group $(\mathrm{O}=\mathrm{C}-\mathrm{NH})$ in crystalline acetamide. J. Mol. Graph. Model. 2008;26:977-981.

26. Davarpanah M, Abbasi H, Nabati M, Sabahnoo H, Bodaghi-Namileh V, Mazidi M, Movahhed-Tazehkand $\mathrm{H}$, M ohammadnejad-M ehrabani $\mathrm{H}$. Kit formulation of active pharmaceutical ingredient $\mathrm{d}, \mathrm{I}$-HM PAO as a brain perfusion diagnostic system. Prog. Chem. Biochem. Res. 2019;2:185-191.

27. Nabati M, Bodaghi-Namileh V, Sarshar S. Molecular modeling of the antagonist compound esketamine and its molecular docking study with non-competitive $\mathrm{N}$ methyl-D-aspartate (NMDA) receptors NR1, NR2A, NR2B and NR2D. Prog. Chem. Biochem. Res. 2019;2:108-119.

28. Nabati M, Bodaghi-Namileh V. Non-competitive Nmethyl-D-aspartate (NM DA) receptor (NR2B) structure in complex with antidepressant arketamine. Iran. J. Org. Chem. 2019;11:2591-2598.

29. Nabati $M$, Bodaghi-Namileh V. Design of novel drugs (P3TZ, H2P3TZ, M 2P3TZ, H4P3TZ and M 4P3TZ) based on zonisamide for autism treatment by binding to potassium voltage-gated channel subfamily $D$ member 2 (Kv4. 2). Int. J. New Chem. 2019;6:254-276.

30. Nabati M, Sabahnoo H. Spectroscopic (FT-IR and UVVis), electronic and docking studies on the red clover isoflavone irilone as a progesterone receptor (PR) effect supporter in endometrial and ovarian cancer cell lines. J. Med. Chem. Sci. 2019;2:118-125.

31. Nabati M. Modeling and interactions analysis of the novel antagonist agent flibanserin with 5hydroxytryptamine $2 \mathrm{~A}(5-\mathrm{HT} 2 \mathrm{~A})$ serotonin receptor as a HSDD treatment in premenopausal women. Iran. Chem. Commun. 2019;7:324-334.

32. Nabati M, Sabahnoo H, Lohrasbi E, M azidi M. Structural Properties Study and Spectroscopic (FT-IR and UV-Vis) Profiling of the Novel Antagonist LY2157299 as a Transforming Growth Factor- $\beta$ (TGF- $\beta$ ) Receptor I Kinase Inhibitor by Quantum-mechanical (QM) and molecular docking techniques. Chem. Method. 2019;3:377-391.

33. Nabati M. Insight into the structural and spectral (IR and UV-Vis) properties of the salts of alkali (Li, $\mathrm{Na}$ and $\mathrm{K})$ and alkaline earth ( $\mathrm{Be}, \mathrm{Mg}$ and $\mathrm{Ca}$ ) metals with pertechnetate oxoanion (99mTc04-) as the convenient water-soluble sources of the radioactive element technetium. Chem. Method. 2019;3:258-270.

34. Nabati M, Bodaghi-Namileh V. Physicochemical properties analysis and dopamine D2 receptor (D2R) docking of zotepine as an atypical antipsychotic antagonist. J. Phys. Theor. Chem. 2018;15:149-157.

35. Nabati M, Bodaghi-Namileh V, Mazidi M. Evaluation of [18F] FPTT molecular structure and its binding to progesterone receptor (PR) for PET scan of breast cancer. J. Phys. Theor. Chem. 2018;15:159-171.

How to cite this article: Nabati M, Pournamdari E, Dashti-Rahmatabadi Y, Sarshar S. Withaferin A (WIT) Interaction with beta-Tubulin to Promote Tubulin Degradation: In Silico Study. Adv. J. Chem. B. 2020;2(1):26-32. doi: 10.33945/SAM I/AJCB.2020.1.5 